\title{
The effects of music intervention on the emotion, haemodynamics and endocrine hormone level of senile patients during the perianesthesia period
}

\author{
Feng Liang, Fang-lei Han and Shuang Qi ${ }^{a}$ \\ China-Japan Union Hospital of Jilin University
}

\begin{abstract}
Purpose: to explore the effects of music intervention on haemodynamics and endocrine hormone level of senile patients. Methods: choosing ASA II -III level and selecting 60 senile patients performed with intraspinal anesthesia; randomly dividing them into the intervention group and control group with 30 cases in each group. Both the two groups of patients completed the SAS before entering the operating room. When the patients of the intervention group entered the operating room, selected music would be played in a continuous loop immediately till the end of the operation while the doctors of the control group just told the patient to relax without music or other special treatment. Recording and comparing the SAS scores, SBP, HR and salivary cortisol concentration of the two groups before and after intervention. Results: the SAS scores, SBP, HR and salivary cortisol concentration of the intervention group were significantly decreased compared with the value before intervention and the differences within and between the groups were significant $(\mathrm{P}<0.05)$. Conclusion: music intervention can effectively reduce the preoperative anxiety, ease intranperative tension and maintain the stability of hemodynamics, so it is beneficial for senile parents to live through the perianesthesia period.
\end{abstract}

Due to the retrogression of organ functions caused by pathological aging, elderly people's response to stress and their adapting mechanism are decreased obviously. Especially for conscious senile patients without general anesthesia in the operation, serious preoperative anxiety and iatrogenic noise caused by psychological stress will lead to excessive excitement of sympathy - LCSA with risks of arrhythmia and sudden death ${ }^{[1]}$. And the excessive activation of hypothalamus - hypophysis cerebri HPA will cause endocrine and immune system disorders, which will significantly affect patients' condition and prognosis ${ }^{[2]}$. Therefore, effective psychological intervention is of great significance for the maintenance of physiological and psychological stability of senile patients during the perioperative period.

Music intervention is one of the ways of mental intervention. It can relieve pain, improve mood and stabilize emotions. It is usually used to assist postoperative rehabilitation and pain therapy clinically ${ }^{[3 \sim 4]}$. But there is less research on its application on senile patients during the perioperative period.

The purpose of this study is to discuss the effects of music intervention on the emotions, haemodynamics and endocrine hormone levels of senile patients so as to provide theoretical basis for its clinical application.

\footnotetext{
a Corresponding author: qishuang@jlu.edu.cn
} 


\section{Materials and Methods}

\section{1 General data}

approved and signed by the Ethics Committee of our hospital, selecting 60 patients performed intraspinal anesthesia during January 2014 to June 2014 in China-Japan union hospital of Jilin University. The patients were $65-75$ years old with unlimited sex. The ASA was at II -III level. The operation was performed around 9 am, lasting 2-3 hours with less amount of bleeding. The patients had no heart, lung, liver, kidney or endocrine system diseases and didn't have serious visual and hearing difficulty or mental illness in the preoperative examination. The ages, income, education degrees and occupations of the two groups had no obvious differences; randomly dividing the patients into intervention group and control group with 30 cases in each group.

\subsection{Research methods}

consultation before the operation day could establish good communication and trust between doctors and patients. Illustrating the effects of music playing systematically and asking patients to select music from alternative music list(hypnotic music) according to their preference. Both the two groups of patients completed SAS before entering the operating room. Then their blood pressure and heart rate got routine monitoring in the operating room. The moment the intervention group entered the operating room, the selected music would be played in a continuous loop until the end of the operation. The voice was controlled in an acceptable range, generally $50 \sim 60 \mathrm{db}$. The patients of the control group were asked to relax without music playing or other special treatment. Try to keep the surroundings quiet.

\subsection{Observing indexes}

(1) emotional state assessment: using SAS of Zung of 1983 to have psychological assessment and then getting an initial score. The initial score multiplying 1.25 equaled to the standard score. The standard score 50 was regarded as the critical value. 50 -59 points were divided into mild degree; 60-69 points were moderate; 70 points or more were severe. The higher the score was, the heavier the anxiety symptom was ${ }^{[6]}$. Recording the SAS scores when entering the operating room (before intervention) and at the end of surgery (after intervention); (2) Hemodynamic assessment: recording the hemodynamic indexes, SBP and HR in the two time points; (3) The endocrine hormone index adopted special salivette. Pouring the sliver in the salivette to patients' mouth for 45-second chewing; then putting the sliver after chewing to the salivette tube. $0.5-1.5 \mathrm{ml}$ saliva could be collected after centrifugation. The saliva was put in $-84^{\circ} \mathrm{C}$ refrigerator for restoration and got detected intensively. Adopting ELISA to measure the concentration of salivary cortisol(kit number: HT81012; place of origin: Canada Pllabs).

\section{4 Statistical analysis}

adopting SPSS 11.0 software to conduct $\chi^{2}$ test to the enumeration data; the measurement data was expressed by mean \pm standard deviation $(\overline{\mathrm{X}} \pm \mathrm{S})$; the comparison within groups was tested by matching $\mathrm{t}$; the comparison between groups was analyzed by one-way variance; $\mathrm{P}<0.05$ meant there existed significant differences.

\section{Results}

2. 1 The comparison of the emotional states (SAS score) of the two groups: before intervention, the SAS scores of the two groups were both over 50 and both the two groups had one case getting more 
than 60 , but there was no significant difference between the two groups $(\mathrm{P}>0.05)$; after intervention, the SAS score of the intervention group declined obviously, so there were significant differences between and within groups $(\mathrm{P}<0.05)$; though the SAS scores of the control group had a rising trend, there was no statistical significance $(\mathrm{P}>0.05)$.

2. 2 The comparison of the haemodynamics of the two groups: the SBP and HR of the two groups had no significant differences ( $\mathrm{P}>0.05)$; After intervention, the SBP and HR of the intervention group declined obviously and the comparison between and within groups had significant difference $(\mathrm{P}<0.05)$ while the control group had no significant change $(\mathrm{P}>0.05)$.

2.3 The comparison of endocrine hormone indicators: before intervention, the saliva cortisol concentration of the two groups had no significant differences $(P>0.05)$; after intervention, the concentration of the intervention group decreased obviously $(\mathrm{P}<0.05)$; while the salivary cortisol concentration of the control group was increased, but there was no statistical significance $(\mathrm{P}>0.05)$. See table 1 .

Table 1. the comparison of the SAS scores, SBP, HR and saliva cortisol concentration of the two groups before and after intervention( $\overline{\mathrm{X}} \pm \mathrm{S}, \mathrm{n}=30)$

\begin{tabular}{|c|c|c|c|}
\hline Groups & Item/time & Before intervention & After intervention \\
\hline \multirow{4}{*}{$\begin{array}{l}\text { The } \\
\text { interven } \\
\text { tion } \\
\text { group }\end{array}$} & SAS scores & $52.20 \pm 7.55$ & $44.23 \pm 3.17^{\Delta \Delta}$ \\
\hline & $\mathrm{SBP}(\mathrm{mmHg})$ & $143.78 \pm 4.52$ & $119.56 \pm 5.65^{\bullet \circ}$ \\
\hline & $\mathrm{HR}(/ \min )$ & $85.21 \pm 3.33$ & $70.17 \pm 6.64^{\star \text { 访 }}$ \\
\hline & $\begin{array}{l}\text { salivary cortisol } \\
\text { (nmol/1) }\end{array}$ & $26.11 \pm 3.45$ & $14.75 \pm 2.12^{\nabla \nabla}$ \\
\hline \multirow{4}{*}{$\begin{array}{l}\text { The } \\
\text { control } \\
\text { group }\end{array}$} & SAS score & $51.10 \pm 7.86$ & $53.60 \pm 7.65$ \\
\hline & $\mathrm{SBP}(\mathrm{mmHg})$ & $144.84 \pm 4.75$ & $145.32 \pm 5.11$ \\
\hline & $\mathrm{HR}(/ \min )$ & $83.70 \pm 3.58$ & $82.71 \pm 3.23$ \\
\hline & $\begin{array}{c}\text { salivary cortisol } \\
(\mathrm{nmol} / \mathrm{l})\end{array}$ & $21.87 \pm 3.53$ & $22.81 \pm 3.55$ \\
\hline
\end{tabular}

After intervention: comparing the SAS scores between groups and within the intervention group, $\mathrm{P}^{\mathbf{\Lambda}}<0.05$; comparing the SBP, $\mathrm{P}^{\bullet}<0.05$; comparing the HR, $\mathrm{P}^{\star}$ 论 $<0.05$; comparing the salivary cortisol, $\mathrm{P}^{\nabla}<0.05$.

\section{Discussions}

As a strong psychological stressor, the fear of surgery and anesthesia has a direct impact on the emotion of perioperative patients. They usually show different degrees of anxiety [7]. Elderly people are easily affected by the recession of physiological functions, chronic diseases, changes of working and living environment, fear of therapeutic effects and other negative events, so their emotions are more likely to fluctuate. This study found that the SAS scores of the two groups before intervention were greater than 50 and both the two groups had a case reaching 60 points(moderate anxiety), which suggested the common occurrence of preoperative anxiety of senile patients; the SAS scores of the control group increased after intervention, which meant that for conscious senile patients, the continuous psychological stress caused by iatrogenic factors (such as the conversation of doctors, noise of surgical instrument and so on) might aggravate the degree of anxiety though relatively perfect anesthesia had been performed.

Intense psychological stress will stimulate hypothalamus - hypophysis cerebri - HPA and sympathetic nerve - LCSA. The excessive cortisol and epinephrine released in the two axis systems 
acts on the body through blood circulation, causing the disorder of haemodynamics and immune system [8]. Elderly people's retrogression of automatic nervous system, poor cardiovascular sympathy/neuroendocrin, gradual retrogression of neurohormone and immune functions as well as intense psychological stress are more likely to cause cardio-cerebral vascular accidents and the occurrence of postoperative complications. The results of this study showed that the SBP, HR and salivary cortisol concentration of the control group increased after intervention while the salivary cortisol concentration of 2 cases reached a critical value after operation (after intervention). It suggested that the maintenance of physical steady-state of elderly people would be increasingly difficult along with the longer lasting time of psychological stress. Therefore, systematic and effective psychological intervention on senile patients during the perianesthesia period is very necessary. Music intervention is a measure of non-invasive intervention with low cost and easy operation. It avoids the influence of pharmacological intervention on patients' cognitive function after operation, so it is more acceptable for patients. The "situationality" and "infectivity" of music can bring patients to a special artistic conception.According to the results of this study, music after the intervention, the SAS scores, SBP, HR and salivary cortisol concentration of the intervention group were all significantly decreased after music intervention, which showed that music intervention could effectively relieve patients' anxiety and reduce the stress response of their bodies. Its mechanism may be: on the one hand, music intervention can alleviate excessive tension of sympathetic nerve, reduce stress reactions and promote emotional sedation [9]; on the other hand, music is acoustic vibration with certain rules. It can be transferred to neural signal via cochlea to cochlear nucleus; then it was conveyed to auditory thalamus and amygdaloid nucleus from the cochlear nucleus; it acts on hypothalamus through the neural pathway between amygdaloid nucleus and hypothalamus to reduce the activity of HPA axis so as to lower the content of cortisol released by adrenal cortex[10]. In this study, patients of the intervention group chose the music they preferred from the alternative music list. It aroused their enthusiasm of participation; in the whole operating process, patients were in beautiful melody, so their emotion was highly relaxed, which fully embodied the development trend of comfortable and humanized medicine.

In a word, music intervention can effectively reduce preoperative anxiety, relieve intranperative tension and maintain the haemodynamics stability of senile patients to help them live through the perianesthesia period safely.

\section{References}

1. Xin-Liang Zhuan, editor. Modern Anesthesiology [M]. Third Edition. Beijing: People's Medical Publishing House, 2003, 190. Chinese.

2. Price MA, Tennant CC, Buttow PN, et al. The role of psychosocial factors in the development of breast carcinoma: Part II . Life event stressor. social support, defense style, and emotional control and their interactions[J].Cancer, 2001;91(4): 686 697.

3. Krishnaswamy, Priyadharshini, et al. Effect of music therapy on pain and anxiety levels of cancer patients: A pilot study[J]Indian Journal of Palliative Care ,2016;22(3) : 307-311

4. Shu-Ming Wang, Lina Kulkarni, Jackqulin Dolev. et a1. Music and Preoperative Anxiety:A Randomized, Controlled Study[J]. Anesth Analg,2003;94:1489 1494.

5. Juan Du. Psychological influence of music on the cesarean section and nursing experience [J]. Journal of Clinical Nursing, 2002;15(1):53-54. Chinese.

6. Nian-Feng Guo, editor. Psychologist (National Occupation Qualification Training Tutorial) [M]. Beijing: National Publishing House, 2002,4. Chinese.

7. Pin-Ling Peng, editor. General Psychology [M]. Beijing: Beijing Normal University Press, 2002,3. Chinese.

8. Natsuaki MN, Klimes-Dougan B, Ge X, Shirtcliff EA,Hastings PD, Zahn-Waxler C. Early pubertal maturation and internalizing problems in adolescence: Sex differences in the role of cortisol reactivity to interpersonalstress[J]. Journal of Clinical Child and Adolescent Psychology, 2009;38(4):513 524. 
9. Wang J, Jian F, Chen J, et al. Cognitive behavioral therapy for orthodontic pain control:A randomized trial[J]. J Dent Res, 2012,91(6):580-585.

10. R. Padmanabhan AJ, Hildreth D, Laws. A prospective, randomised, controlled study examining binaural beat audio and preoperative anxiety in patients undergoing general anaesthesia for day case surgery[J].Anaesthesia,2005;60:874 877. 